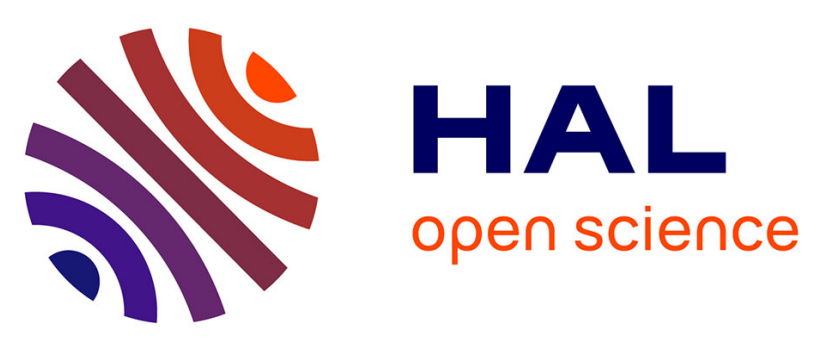

\title{
Concerted action of two novel tRNA mtDNA point mutations in chronic progressive external ophthalmoplegia
}

Cornelia Kornblum, Gábor Zsurka, Rudolf J. Wiesner, Rolf Schröder, Wolfram S. Kunz

\section{To cite this version:}

Cornelia Kornblum, Gábor Zsurka, Rudolf J. Wiesner, Rolf Schröder, Wolfram S. Kunz. Concerted action of two novel tRNA mtDNA point mutations in chronic progressive external ophthalmoplegia. Bioscience Reports, 2008, 28 (2), pp.89-96. 10.1042/BSR20080004 · hal-00479296

\section{HAL Id: hal-00479296 https://hal.science/hal-00479296}

Submitted on 30 Apr 2010

HAL is a multi-disciplinary open access archive for the deposit and dissemination of scientific research documents, whether they are published or not. The documents may come from teaching and research institutions in France or abroad, or from public or private research centers.
L'archive ouverte pluridisciplinaire HAL, est destinée au dépôt et à la diffusion de documents scientifiques de niveau recherche, publiés ou non, émanant des établissements d'enseignement et de recherche français ou étrangers, des laboratoires publics ou privés. 


\section{Concerted action of two novel tRNA mtDNA point mutations in chronic progressive external ophthalmoplegia}

Cornelia Kornblum ${ }^{a}$, Gábor Zsurka ${ }^{\text {b, c }}$, Rudolf J. Wiesner ${ }^{\text {d, e }}$, Rolf Schröder ${ }^{f, g}$, Wolfram S. Kunz*b,c

${ }^{a}$ Department of Neurology, University of Bonn Medical Center, D-53105 Bonn, Germany

${ }^{\mathrm{b}}$ Department of Epileptology, University of Bonn Medical Center, D-53105 Bonn, Germany

${ }^{c}$ Platform NeuroCognition, Life \& Brain Center, D-53105 Bonn, Germany

${ }^{d}$ Institute of Vegetative Physiology, Faculty of Medicine, University of Köln, D-50931 Köln, Germany

${ }^{\text {e }}$ Center for Molecular Medicine Cologne (CMMC), University of Köln, D-50931 Köln, Germany

${ }^{f}$ Department of Neuropathology, University of Erlangen, D-91054 Erlangen, Germany

${ }^{9}$ Department of Neurology, University of Erlangen, D-91054 Erlangen, Germany

\section{Running title}

Concerted action of tRNA mtDNA mutations in CPEO

\section{*Correspondence to:}

Prof. Dr. Wolfram S. Kunz, NeuroCognition, Life \& Brain Center, University of Bonn, Sigmund-Freud-Str. 25, D-53105 Bonn, Germany. Tel: +49 2286885290; Fax: +49 2286885295; E-mail: wolfram.kunz@ukb.uni-bonn.de 


\section{Synopsis}

Chronic progressive external ophthalmoplegia (CPEO) ${ }^{1}$ is a common mitochondrial disease phenotype in adults which is due to mitochondrial DNA (mtDNA) point mutations in a subset of patients. Attributing pathogenicity to novel tRNA mtDNA mutations still poses a challenge particularly when several mtDNA sequence variants are present. Here we report on a CPEO patient in whom sequencing of the mitochondrial genome revealed three novel tRNA mtDNA mutations: G5835A, del4315, T1658C in tRNA ${ }^{\text {Tyr }}$, tRNA ${ }^{\text {lle }}$, and tRNA ${ }^{\text {Val }}$ genes. In skeletal muscle, tRNA ${ }^{\mathrm{Val}}$ and tRNA ${ }^{\text {Ile }}$ mutations were homoplasmic, while the tRNA ${ }^{\text {Tyr }}$ mutation was heteroplasmic. To address the pathogenic relevance, we performed two types of functional tests: (i) single skeletal muscle fiber analysis comparing G5835A mutation loads and biochemical phenotypes of corresponding fibers, (ii) Northern blot analyses of mitochondrial tRNA $^{\text {Tyr }}$, tRNA ${ }^{\text {lle }}$, tRNA ${ }^{\text {Val }}$. We demonstrated that both the G5835A tRNA ${ }^{\text {Tyr }}$ and del4315A tRNA ${ }^{\mathrm{Il}}$ mutation have serious functional consequences. Single fiber analyses displayed a high threshold of the tRNA ${ }^{\text {Tyr }}$ mutation load for biochemical phenotypic expression on the single cell level indicating a rather mild pathogenic effect. In contrast, skeletal muscle tissue showed a severe decrease in respiratory chain activities, a reduced overall cytochrome $c$ oxidase (COX) staining intensity, and abundant COX-negative fibers. Northern blot analyses showed a dramatic reduction of $\mathrm{tRNA}^{\mathrm{Tyr}}$ and $t R N A^{\text {lle }}$ levels in muscle with impaired charging of tRNA $^{\text {lle }}$, whereas tRNA ${ }^{\mathrm{Val}}$ levels were only slightly decreased with aminoacylation unaffected. Our findings suggest that the heteroplasmic tRNA ${ }^{\text {Tyr }}$ and homoplasmic tRNA ${ }^{\text {lle }}$ mutation act together resulting in a concerted effect on the biochemical and histological phenotype. Thus, homoplasmic mutations may influence the functional consequences of pathogenic heteroplasmic mtDNA mutations.

\section{Keywords}

mitochondrial DNA, chronic progressive external ophthalmoplegia, mitochondrial tRNA, oxidative phosphorylation, mitochondrial disorders, mitochondrial DNA diseases

\section{${ }^{1}$ List of abbreviations}

CPEO - chronic progressive external ophthalmoplegia

mtDNA - mitochondrial DNA

A - adenine

$\mathrm{G}$ - guanine

C - cytosine

$\mathrm{T}$ - thymine

$U$ - uracil

lle - isoleucine

$\mathrm{Val}$ - valine

Tyr - tyrosine

Leu - leucine

tRNA - transfer RNA

MELAS - mitochondrial encephalomyopathy with lactic acidosis and stroke-like episodes

COX - cytochrome coxidase

$\mathrm{SDH}$ - succinate dehydrogenase

CS - citrate synthase

$\mathrm{PCR}$ - polymerase chain reaction

RFLP - restriction fragment length polymorphism 


\section{Introduction}

Chronic progressive external ophthalmoplegia (CPEO) is one of the most common mitochondrial disease phenotypes in adults. In approximately $50 \%$ of CPEO patients, mitochondrial DNA (mtDNA) analysis of skeletal muscle tissue reveals single large-scale mtDNA deletions that usually arise sporadically [1]. However, CPEO may as well be due to maternally inherited or sporadic mtDNA point mutations, or to mutations in nuclear genes resulting in autosomal disorders of intergenomic communication [2,3]. The identification of the specific pathogenic disease-defining mtDNA point mutation often remains a challenge, particularly when several mtDNA sequence variants are present. Due to the high variability of the mitochondrial genome some earlier established criteria for pathogenicity of mtDNA mutations, e.g. the absence in a control population, might fail in cases of previously not described "private" mtDNA mutations. Moreover, even heteroplasmy is no longer a clear criterion for pathogenicity since many heteroplasmic polymorphisms have been reported [4], and various homoplasmic pathogenic tRNA mtDNA point mutations have been identified to date [5-9]. 


\section{Materials and Methods}

\section{Patients}

A 22-year-old male patient of Turkish ethnic origin (patient $A$ ) presented with a medical history of slowly progressive upper eye lid ptosis first realized at the age of 15 years. Later, he developed incomplete external ophthalmoplegia as well as exercise intolerance. His further past medical history was uneventful. All family members lived in a secluded village in the province of Sakarya (Adapazari), Eastern Turkey, and were reported to be free of neuromuscular symptoms. The patient underwent a neurological investigation, open skeletal muscle biopsy including histochemical, biochemical, and molecular genetic investigations as well as genetic analyses of leukocyte and buccal mucosa DNA. Blood for genetic analysis was obtained from all accessible family members (mother, two sisters, and one brother).

To compare functional features of mtDNA mutations in patient $A$, we obtained corresponding histological and biochemical data from patient $B$ (female, aged 37 years at time of open skeletal muscle biopsy) presenting with mitochondrial encephalomyopathy with lactic acidosis and stroke-like episodes (MELAS) due to the tRNA ${ }^{\text {Leu(UUR) }}$ A3243G mtDNA point mutation. In patient B, only skeletal muscle tissue was available for analysis. Skeletal muscle biopsies from 43 neurologically unaffected patients ( 25 male, 18 female, age range 3-77 years) and no histologic evidence for a neuromuscular disorder were used as controls.

All examinations were conducted according to the Declaration of Helsinki (2000) of the World Medical Association and to guidelines of the local ethics committee of the University of Bonn. Informed consent was obtained from all investigated patients.

\section{Skeletal muscle histology}

$6 \mu \mathrm{m}$ consecutive cryostate sections of open vastus lateralis muscle biopsies were processed for standard histologic and enzyme-histochemical work-up as previously described [10]. Quantitative histochemical single fiber analyses of cytochrome $c$ oxidase $(\mathrm{COX})$ and succinate dehydrogenase (SDH) activities were performed using fiber specific grey level determination of 12-bit video images in patient $A[11,12]$.

\section{Biochemical investigations}

Respiratory chain enzyme activities [rotenone sensitive NADH:CoQ1 oxidoreductase (complex I), COX (complex IV)], and citrate synthase (CS) in skeletal muscle homogenate were measured spectrophotometrically as previously described [13]. Results were given in $\mathrm{U} / \mathrm{g}$ wet weight and were corrected for potential fiber type variations or adaptive mitochondrial proliferation by normalization of data for the mitochondrial matrix marker enzyme CS.

\section{Molecular genetic analyses}

We extracted genomic DNA from about $30 \mathrm{mg}$ skeletal muscle specimens and from buccal mucosa with the QiaAmp DNA Mini Kit (Qiagen, Hilden, Germany). Genomic DNA was isolated from $10 \mathrm{ml}$ aliquots of EDTA anticoagulated blood using a salting out method [14]. MtDNA was screened for large-scale rearrangements by Southern blotting of Pvull-cleaved total DNA from skeletal muscle using digoxigenin-labeled isolated human mtDNA as full length mtDNA probe (all restriction endonucleases from New England Biolabs, Frankfurt, Germany). The presence of duplications was excluded by digestion with Pmel and BamHI. For direct sequence analysis, 28 overlapping PCR fragments were amplified from the whole mitochondrial genome of skeletal muscle DNA and sequenced using a commercial sequencing service (MWG Biotech, Ebersberg, Germany).

To quantitate A3243G and G5835A mutation loads, we used different polymerase chain reaction (PCR)-restriction fragment length polymorphism (RFLP) assays. For quantification of G5835A, we used a forward primer 5'-GCACCCTAATCAACTGGCTTC-3', and a reverse 
mismatch primer (mismatch underlined) 5'-CTAAAGACAGGGGTTAGGGCT-3' to introduce a novel Banll restriction site in the wild-type allele. To introduce a novel $\bar{B}$ ceAl restriction site in the mutant allele, we designed a forward primer 5'-AGCTAAGCACCCTAATCAACTG-3', and a mismatch reverse primer with a T7-extension 5'-

TAATACGACTCACTATAGGGAAATCTAAAGACAGGGGTTAGGCCG-3' (italic, T7extension). For quantification of $A 3243 \mathrm{G}$, a PCR product was amplified using a forward primer 5'-GAAAGGACAAGAGAAATAAGGCCT-3', and a reverse primer, 5'GGAATTGAACCTCTGACTGTAAAG-3'. PCR products were digested with Haell

Amplification conditions were as follows: $95^{\circ} \mathrm{C}$ for $10 \mathrm{~min} ; 33$ cycles of $95^{\circ} \mathrm{C}$ for $15 \mathrm{~s}, 55^{\circ} \mathrm{C}$ for $30 \mathrm{~s}$, and $72^{\circ} \mathrm{C}$ for $40 \mathrm{~s}$; and finally $72^{\circ} \mathrm{C}$ for $7 \mathrm{~min}$. We separated restriction fragments on $10 \%$ polyacrylamide gels and visualized them by SYBR Green I staining (Sigma-Aldrich, Steinheim, Germany). The proportions of mutant and wild-type mtDNA were calculated from band intensities with local area background subtraction (Image $\mathrm{J}$ analysis software, rsb.info.nih.gov/ij).

\section{Single fiber PCR analysis}

We used $10 \mu \mathrm{m}$ sections of unstained frozen skeletal muscie tissue of patient A located inbetween the muscle sections stained for $\mathrm{COX}$ and $\mathrm{SDH}$ activity. Fiber types of single fibers were distinguished based on myosin ATPase stainings at $\mathrm{pH} 4.6$ and 9.4. For single fiber PCR analysis, we randomly selected 20 type I fibers with comparable SDH activity quantified by fiber specific grey level determination to examine the dependency of the cellular biochemical function on the mutation load. Laser microdissection of muscle fibers was performed as previously described [15]. To determine the G5835A mutation load in single fibers, we used a nested PCR technique. First, we amplified a PCR product spanning the mutation site using the forward primer 5'-CCTTACCACGCTACTCCTACCTATCTCC-3' (nucleotides 5462-5489), and the reverse primer 5'-CTCCAGCTCATGCGCCGAATAATAG3' (nucleotides 5985-5961). In the second round of nested PCR, we subjected PCR products of the first round to mismatch PCR-RFLP with digestion of the wild-type allele as described above.

\section{Northern Blot}

Total RNA was isolated from a small piece of skeletal muscle tissue of patient A, $30-50 \mathrm{mg}$ pieces of muscle tissue of four healthy control subjects, and cultured human osteosarcoma cells (143B cell line). To maintain charging of the tRNAs with amino acid and separate tRNA from aminoacylated tRNA, RNA was isolated and electrophoresed under acidic conditions on long gels as previously described in detail [16]. Regions of mtDNA encompassing mitochondrial tRNA genes were amplified from 143B cell DNA by PCR and used as probes. For each tRNA, two different probes were used, either specifically designed for wild-type or mutant tRNA. Primer sequences used for the design of tRNA ${ }^{\text {Leu(UUR) }}$, tRNA ${ }^{\mathrm{Val}}$, tRNA ${ }^{\text {Tyr }}$, and tRNA ${ }^{\text {Ile }}$ probes can be obtained from the corresponding author by request. For the normalisation of tRNAs to a non-mitochondrial small RNA species, blots were hybridised to a 5 S rRNA probe (5'-GGGTGGTATGGCCGTAGAC-3') endlabeled using ( $\left.\mathrm{y}^{-{ }^{32}} \mathrm{P}\right)$ ATP (5000 $\mathrm{Ci} / \mathrm{mmol}$, Hartmann Analytic, Braunschweig, Germany) and T4-PNK [17]. Purified PCR products (QIAquick PCR purification columns, Qiagen, Hilden, Germany) were radiolabeled with ( $\alpha^{-32}$ P)-dCTP (5000 Ci/mmol; Hartmann Analytic, Braunschweig, Germany) by the random-primer method, and unincorporated nucleotides were removed from labeling reactions by gel filtration through ChromaSpin columns (Clontech, Heidelberg, Germany). Prehybridisation, hybridisation and analysis of the data by phosphoimaging was carried out as described [16]. The two bands corresponding to aminoacylated and non-acylated tRNA were identified by comparison of native to alkali-treated, deacylated RNA samples from 143B cells and quantitated individually in order to estimate the degree of loading with amino acid. Finally, the sum of both values was divided by the signal for 5S rRNA to obtain tissue levels of tRNAs. 


\section{Results}

\section{Histochemical and biochemical evaluation of skeletal muscle shows an exceptionally severe mitochondrial pathology}

As shown in Fig. 1, back-to-back stained muscle biopsy sections from patient $A$ revealed a weaker overall COX staining, abundant SDH-positive COX-negative fibers (indicated by circles, "mosaic" COX staining pattern), and ragged red fibers (stars) in comparison to patient $B$ with a similar mutation load of the heteroplasmic A3243G mtDNA point mutation (Fig. 1 b, e). The low overall COX staining of muscle fibers from patient $A$ is not a result of a lowered mitochondrial content as evidenced from the SDH staining intensities in Figs. 1c and $\mathbf{f}$. Mitochondrial respiratory chain enzyme activities in skeletal muscle homogenate of patient $A$ were compared to controls and to patient B (Table 1). In line with the histological stainings the data showed a dramatic decline of absolute activities of complex I and complex IV in the biopsy of patient $A$, but also when normalized for CS activity.

\section{MtDNA sequence analysis reveals three novel and potentially pathogenic tRNA mtDNA point mutations}

MtDNA large-scale rearrangements were ruled out. Direct sequence analysis of the mitochondrial genome isolated from skeletal muscle tissue of patient $A$ revealed three novel and putative pathogenic tRNA mtDNA point mutations among a multitude of mutations when compared to the reference sequence [18]. For mutation details, please refer to Fig. 2.

a) We identified a novel $G$ to $A$ exchange at position 5835 of mtDNA which was found to be heteroplasmic in skeletal muscle. The G5835A mutation is located in the mitochondrial tRNA ${ }^{\text {Tyr }}$ gene and causes a $\mathrm{C} \rightarrow U$ transition at an evolutionary highly conserved position in the T-stem of the tRNA ${ }^{\text {Tyr }}$ (Fig. 2a). The mutation has not been previously described in human mtDNA databases and is not present in more than 2500 controls (cf. MITOMAP, www.mitomap.org; Human Mitochondrial Genome Database, www.genpat.uu.se/mtDB).

b) We also detected a previously not described homoplasmic mutation, del4315A, in the mitochondrial tRNA ${ }^{\text {lle }}$ gene affecting the tRNA T $\psi$ C loop (Fig. 2b).

c) Finally, a not yet reported homoplasmic $U \rightarrow C$ transition was detected at position 1658 in the mitochondrial tRNA ${ }^{\text {Val }}$ gene affecting the tRNA T-stem at an evolutionary not well conserved position (Fig. 2c). Further sequencing revealed two novel homoplasmic mutations in protein-coding genes: a C11972T transition in the ND4 gene, and a A14539G transition in the ND6 gene (cf. MITOMAP; Human Mitochondrial Genome Database), both genes encoding subunits of complex I. With high probability C11972T and A14539G are without functional consequences since they do not result in amino acid changes. A known heteroplasmic $D$-loop polymorphism was detected at position 72 resulting in a $T \rightarrow C$ transition in skeletal muscle mtDNA. Sequencing revealed the following homoplasmic polymorphisms being in line with the mitochondrial haplogroup "H": 73A, 2706A, 7028C. More than 400 of the publicly available complete mitochondrial coding region sequences belong to haplogroup "H". None of the five novel mutations mentioned above has been reported in these phylogenetically related individuals, or in more than 2100 other, not related sequences. The further identified polymorphisms C64T, A263G, ins310C, A750G, A1438G, A4769G, A8860G, A15326G, A16269G, and T16519C did not allow a more precise classification of the sample to the subgroups of the "H" haplogroup so far described [22].

Mismatch PCR-RFLP analysis demonstrated in accordance with the sequence analysis (Fig. 3a) the presence of G5835A in $69 \pm 3 \%$ of skeletal muscle bulk mtDNA in patient A (Figs. $3 b$, c, lane 2). This mutation was restricted to muscle and not present in buccal mucosa and blood (Figs. 3b, c, lanes 3-4). Analysis of blood samples of two sisters, one brother, and the patient's mother did not reveal detectable amounts of the heteroplasmic mutation (Figs. 3b, c, lanes 5-8). These findings suggest a mutational event after germ-layer differentiation, however a mutation arisen early in embryogenesis or in the maternal germ line cannot be 
excluded. All other mutations were found to be homoplasmic in the blood of the patient, one sister, and their mother (other relatives were not analysed for these mutations).

Skeletal muscle single fiber PCR analysis in patient A showed that all SDH-positive COXnegative fibers as evidenced by comparison of COX-and SDH-activity (Fig. 1b, c) harboured at least $98 \%$ of the G5835A mutation, which demonstrates a high threshold for phenotypical expression on the single cell level (Fig. 4).

\section{The levels of the mitochondrial tRNAs for tyrosine and isoleucine are severely reduced}

In comparison to the nuclear encoded 5 S rRNA, which served as a loading control, we observed a $95 \%$ reduction of the tRNA ${ }^{\text {Tyr }}$ amount in total skeletal muscle RNA of patient $A$ (Fig. 5a, b). tRNA ${ }^{\text {lle }}$ levels were found to be decreased by about $85 \%$. In contrast, the tRNA ${ }^{\mathrm{Val}}$ amount was reduced by only about $60 \%$. The levels of $t R N A^{\text {Leu(UUR) }}$ were even slightly increased. The same results were independently obtained for each tRNA with two different probes designed for wild-type (Fig. 5a) or mutant tRNA (data not shown). This approach rules out the possibility that lower tRNA signals in the patient's muscle might be caused by poorer hybridization of wild-type probes to mutant tRNAs. Evaluation of the ratio of aminoacylated vs. non-acylated tRNA showed that tRNA ${ }^{\text {Tyr }}$ and tRNA ${ }^{\text {Val }}$ were predominantly loaded with amino acid in control muscles, while tRNA ${ }^{\text {lle }}$ and tRNA ${ }^{\text {Leu(UUR) }}$ were equally present in acylated and deacylated forms. As shown in Fig. 5a, charging of tRNA ${ }^{\text {lle }}$ with amino acids was clearly impaired in the patient's muscle, with less than $15 \%$ compared to app. $40 \%$ in controls.

\section{Discussion}

The diagnosis of mitochondrial disorders often requires a complex approach including clinical evaluation, skeletal muscle histology, biochemical and finally molecular genetic analyses [23]. However, assigning pathogenicity to mtDNA point mutations is often a challenge $[24,25]$, especially when novel mutations are present in individuals with an ethnical background that has not been extensively studied with respect to haplogroup defining mutations. Moreover, since various homoplasmic tRNA mtDNA point mutations have been proved to be pathogenic [5-9] and many apparently neutral polymorphisms are heteroplasmic [4], the heteroplasmic state of a mutation is no longer a major criterion for pathogenicity. Equally problematic is the criterion of degree of conservation among species, since e.g. the A3243G tRNA ${ }^{\text {Leu(UUR) }}$ mtDNA mutation with proven pathogenicity in humans is the normal genotype in Canis familiaris.

This diagnostic challenge is amply illustrated in the genetic analysis of our CPEO patient in whom we detected five novel mtDNA mutations. Two mutations were located in proteincoding genes and were silent mutations, not changing amino acids. However, all further three novel tRNA mtDNA point mutations are potentially pathogenic. To address the pathogenic relevance of each of these mutations, we performed (i) single skeletal muscle fiber analysis, and (ii) Northern blot analyses as functional tests.

Demonstrating a correlation between biochemical phenotype and mutation loads of heteroplasmic mtDNA point mutations in individual cells has been long used as a strong evidence for pathogenicity [25]. In our patient, we found that those muscle fibers that displayed a failure of oxidative phosphorylation (as indicated by negative COX staining in $\mathrm{SDH}$-positive fibers) harboured very high levels of the heteroplasmic G5835A tRNA ${ }^{\mathrm{Tyr}}$ mutation. Thus, the heteroplasmic tRNA ${ }^{\text {Tyr }}$ mutation is proven to be functionally relevant and plays a primary role in creating the mosaic pattern in our histochemical COX muscle stainings. Its $98 \%$ mutation load threshold for cellular biochemical impairment is however higher than described for the A3243G tRNA ${ }^{\text {Leu(UUR) }}$ mutation [26] and the G12276A tRNA ${ }^{\text {Leu(CUN) }}$ mtDNA mutation [12,27], which suggests a mild functional effect of the mutation. This contrasts to the severe biochemical alterations present in the patient's skeletal muscle homogenate (Table 1) and to the histological findings with reduced overall COX staining 
intensity, a mosaic pattern of COX activity, and abundant ragged red fibers (Fig. 1). The biochemical phenotype and general decrease in COX activity (including fibers with low G5835A mutation loads) apart from the mosaic pattern cannot be explained by the presence of the heteroplasmic G5835A tRNA ${ }^{\text {Tyr }}$ mtDNA mutation alone. Functional analyses of both further tRNA mtDNA mutations demonstrated that the tRNA ${ }^{\text {lle }}$ mutation plays an addition role for pathogenicity. Since this mutation is homoplasmic, it affects all cells, which readily explains the overall decrease of the COX staining in skeletal muscle biopsy. In this respect, Northern blot analyses revealed that apart from tRNA ${ }^{\text {Tyr }}$, also tRNA ${ }^{\text {val }}$ and tRNA ${ }^{\text {lle }}$ showed reduced steady state levels, leaving a remaining $40 \%$ and $15 \%$ of the respective tRNAs as compared to controls. The latter value is similar to residual tRNA amounts reported in previous studies on homoplasmic pathogenic tRNA mtDNA mutations $(9-15 \%$ and $5-10 \%$, respectively [6,7]). Impaired aminoacylation of tRNA ${ }^{\text {lle }}$ was also observed further stressing the pathogenic relevance. However, the presence of the homoplasmic tRNA ${ }^{\text {lie }}$ mutation alone appears not to cause serious clinical symptoms. The patient's maternal relatives carried all homoplasmic mutations present in the patient, but no family member showed apparent signs of a neurological disorder. We suggest that the G5835A tRNA ${ }^{\text {Tyr }}$ and del4315A tRNA ${ }^{\text {lle }}$ mutation act in concert and perform a "double trouble" of translation of mitochondrially encoded proteins. Since Tyr and lle codons are present at high frequencies in most protein coding mitochondrial genes, low levels of these tRNAs in combination with a defect in charging of tRNA ${ }^{\text {lle }}$ with amino acids readily explain the severe biochemical and histological phenotype. In contrast, the $60 \%$ reduction of tRNA ${ }^{\mathrm{Val}}$ is probably not sufficient to contribute to an additional impairment of translation. This interpretation is in line with the conservation of sites among species - the tRNA ${ }^{\text {Tyr }}$ mutation is located at a strictly conserved site, the tRNA ${ }^{\text {lle }}$ mutation is located at a site which is conserved among primates, while the tRNA ${ }^{\text {Val }}$ mutation occurred at a non-conserved site. Not surprising, Northern blot analysis confirmed the considerable pathogenic role of the heteroplasmic G5835A tRNA ${ }^{\text {Tyr }}$ mutation since tRNA ${ }^{\text {Tyr }}$ steady state levels were found to be decreased by $95 \%$. This is higher than the skeletal muscle bulk mtDNA mutation load which might indicate an effect of mutant tRNAs on the stability of wild-type tRNAs. Interaction between tRNA molecules can be a possible mechanism for this effect and has been previously described for dimerized A3243G tRNA ${ }^{\text {Leu(UUR) }}[28]$.

In conclusion, we demonstrated that two of five not previously described mtDNA mutations, the G5835A tRNA ${ }^{\text {Tyr }}$ and del4315A tRNA ${ }^{\text {lle }}$ mutation, are functionally relevant. For both mutations, this was proven by Northern blot analyses, for the heteroplasmic mutation by single fiber analysis as well. Single fiber data suggested a rather mild effect of the G5835A tRNA $^{\mathrm{Tyr}}$ mutation, which was in contrast to severe histological and biochemical skeletal muscle findings. Concerted pathogenic effects of both mutations on the skeletal muscle phenotype can readily explain these findings. Our study underlines the importance of assessment of both (i) the complete sequence information, and (ii) the biochemical effects of each candidate pathogenic mutation in this process. The interaction of tRNA mtDNA point mutations illustrated here provides a basis for understanding how homoplasmic mutations, specific for a family or even an entire haplogroup, may influence the functional effects of pathogenic heteroplasmic mtDNA point mutations. 


\section{Acknowledgements}

This study was supported by the Deutsche Forschungsgemeinschaft (KU-911/15-1, SCHR562/4-3) and the Center for Molecular Medicine Cologne (CMMC, C9). The skilful technical assistance of Maria Bust (Cologne), Ulrike Strube and Karin Kappes-Horn (Bonn) is gratefully acknowledged. We wish to thank the patient and family members in Sakarya (Adapazari), Eastern Turkey, for their cooperation.

\section{Note}

Nucleotide sequence data reported are available in the DDBJ/EMBL/GenBank databases under the accession number DQ473537 


\section{References}

[1] Biousse, V. and Newman, N.J. (2003) Neuro-ophthalmology of mitochondrial diseases. Curr. Opin. Neurol. 16, 35-43

[2] Hirano, M. and DiMauro, S. (2001) ANT1, Twinkle, POLG, and TP: new genes open our eyes to ophthalmoplegia. Neurology 57, 2163-2165

[3] Mancuso, M., Filosto, M., Choub, A., Tentorio, M., Broglio, L., Padovani, A. and Siciliano, G. (2007) Mitochondrial DNA-related disorders. Biosci. Rep. 27, 31-37

[4] Tully, L.A., Parsons, T.J., Steighner, R.J., Holland, M.M., Marino, M.A. and Prenger, V.L. (2000) A sensitive denaturing gradient-gel electrophoresis assay reveals a high frequency of heteroplasmy in hypervariable region 1 of the human mtDNA control region. Am. J. Hum. Genet. 67, 432-443

[5] McFarland, R., Clark, K.M., Morris, A.A., Taylo,r R.W., Macphail, S., Lightowlers, R.N., and Turnbull, D.M (2002) Multiple neonatal deaths due to a homoplasmic mitochondrial DNA mutation. Nat. Genet. 30, 145-146

[6] McFarland, R., Schaefer, A.M., Gardner, J.L., Lynn, S., Hayes, C.M., Barron, M.J., Walker, M., Chinnery, P.F., Taylor, R.W. and Turnbull, D.M. (2004) Familial myopathy: new insights into the T14709C mitochondrial tRNA mutation. Ann. Neurol. 55, 478-484

[7] Taylor, R.W., Giordano, C., Davidson, M.M., d'Amati, G., Bain, H., Hayes, C.M., Leonard, H., Barron, M.J., Casali, C., Santorelli, F.M., Hirano, M., Lightowlers, R.N., DiMauro, S., and Turnbull, D.M. (2003) A homoplasmic mitochondrial transfer ribonucleic acid mutation as a cause of maternally inherited hypertrophic cardiomyopathy. J. Am. Coll. Cardiol. 41, 1786-1796

[8] Limongelli, A., Schaefer, J., Jackson, S., Invernizzi, F., Kirino, Y., Suzuki, T., Reichmann, H. and Zeviani, M. (2004) Variable penetrance of a familial progressive necrotising encephalopathy due to a novel tRNA(lie) homoplasmic mutation in the mitochondrial genome. J. Med. Genet. 41, 342-349

[9] Scuderi, C., Borgione, E., Musumeci, S., Elia, M., Castello, F., Fichera, M., Davidzon, G. and DiMauro, S. (2007) Severe encephalomyopathy in a patient with homoplasmic A5814G point mutation in mitochondrial tRNACys gene. Neuromuscul. Disord. 17, 258261

[10] Dubowitz, V. and Sewry, C.A. (2006) Muscle biopsy, a practical approach. $3^{\text {rd }}$ edn. W.B. Saunders, Elsevier

[11] Varlamov, D.A., Kudin, A.P., Vielhaber, S., Schröder, R., Sassen, R., Becker, A., Kunz, D., Haug, K., Rebstock, J., Heils, A., Elger, C.E. and Kunz, W.S. (2002) Metabolic consequences of a novel missense mutation of the mtDNA CO I gene. Hum. Mol. Genet. $11,1797-1805$

[12] Zsurka, G., Schröder, R., Kornblum, C., Rudolph, J., Wiesner, R.J., Elger, C.E. and Kunz, W.S. (2004) Tissue dependent co-segregation of the novel pathogenic G12276A mitochondrial tRNALeu(CUN) mutation with the A185G D-loop polymorphism. J. Med. Genet. 41, e124

[13] Wiedemann, F.R., Vielhaber, S., Schröder, R., Elger, C.E. and Kunz, W.S. (2000) Evaluation of methods for the determination of mitochondrial respiratory chain enzyme activities in human skeletal muscle samples. Anal. Biochem. 279, 55-60

[14] Miller, S.A., Dykes, D.D. and Polesky, H.F. (1988) A simple salting out procedure for extracting DNA from human nucleated cells. Nucleic Acids Res. 16, 1215

[15] Zsurka, G., Kraytsberg, Y., Kudina, T., Kornblum, C., Elger, C.E., Khrapko, K. and Kunz, W.S. (2005) Recombination of mitochondrial DNA in skeletal muscle of individuals with multiple mitochondrial DNA heteroplasmy. Nat. Genet. 37, 873-877

[16] Maniura-Weber, K., Helm, M., Engemann, K., Eckertz, S., Möllers, M., Schauen, M., Hayrapetyan, A., von Kleist-Retzow, J.C., Lightowlers, R.N., Bindoff, L.A. and Wiesner, 
J.R. (2006) Molecular dysfunction associated with the human mitochondrial 3302A $>G$ mutation in the MTTL1 (mt-tRNA ${ }^{\text {Leu(UUR) }}$ ) gene. Nucleic Acids Res. 34, 6404-6415

[17] Yasukawa, T., Hino, N., Suzuki, T., Watanabe, K., Ueda, T. and Ohta, S. (2000) A pathogenic point mutation reduces stability of mitochondrial mutant tRNA(Ile). Nucleic Acids Res. 28, 3779-3784

[18] Andrews, R.M., Kubacka, I., Chinnery, P.F., Lightowlers, R.N., Turnbull, D.M. and Howell, N. (1999) Reanalysis and revision of the Cambridge reference sequence for human mitochondrial DNA. Nat. Genet. 23, 147

[19] Sprinzl, M., Horn, C., Brown, M., loudovitch, A. and Steinberg, S. (1998) Compilation of tRNA sequences and sequences of tRNA genes. Nucleic Acids Res. 26, 148-153

[20] Helm, M., Brule, H., Friede, D., Giege, R., Putz, D. and Florentz, C. (2000) Search for characteristic structural features of mammalian mitochondrial tRNAs. RNA 6, 13561379

[21] Pruitt, K.D., Tatusova, T. and Maglott, D.R. (2005) NCBI Reference Sequence (RefSeq): a curated non-redundant sequence database of genomes, transcripts and proteins. Nucleic Acids Res. 33, D501-504

[22] Achilli, A., Rengo, C., Magri, C., Battaglia, V., Olivieri, A., Scozzari, R., Cruciani, F., Zeviani, M., Briem, E., Carelli, V., Moral, P., Dugoujon, J.M., Roostalu, U., Loogvali, E.L., Kivisild, T., Bandelt, H.J., Richards, M., Villems, R., Santachiara-Benerecetti, A.S., Semino, O. and Torroni, A. (2004) The molecular dissection of mtDNA haplogroup H confirms that the Franco-Cantabrian glacial refuge was a major source for the European gene pool. Am. J. Hum. Genet. 75, 910-918

[23] Siciliano, G., Volpi, L., Piazza, S., Ricci, G., Mancuso, M. and Murri, L. (2007) Functional diagnostics in mitochondrial diseases. Biosci. Rep. 27, 53-67

[24] DiMauro, S. and Schon, E.A. (2001) Mitochondrial DNA mutations in human disease. Am. J. Med. Genet. 106, 18-26

[25] McFarland, R., Elson, J.L., Taylor, R.W., Howell, N. and Turnbull, D.M. (2004) Assigning pathogenicity to mitochondrial tRNA mutations: when "definitely maybe" is not good enough. Trends Genet. 20, 591-596

[26] Petruzzella, V., Moraes, C.T., Sano, M.C., Bonilla, E., DiMauro, S. and Schon, A.E. (1994) Extremely high levels of mutant mtDNAs co-localize with cytochrome c oxidasenegative ragged-red fibers in patients harboring a point mutation at nt 3243 . Hum. Mol. Genet. 3, 449-454

[27] Cardaioli, E., Da Pozzo, P., Radi, E., Dotti, M.T. and Federico, A. (2005) A novel heteroplasmic tRNA(Leu(CUN)) mtDNA point mutation associated with chronic progressive external ophthalmoplegia. Biochem. Biophys. Res. Commun. 327, 675-678

[28] Wittenhagen, L.M. and Kelly, S.O. (2002) Dimerization of a pathogenic human mitochondrial tRNA Nat. Struct. Biol. 9, 586-590 


\section{Table 1}

Mitochondrial respiratory chain enzyme activities in skeletal muscle homogenate.

\section{Respiratory chain enzyme} activity in $\mathrm{U} / \mathrm{g}$ wwt

\section{controls}

$(\mathrm{N}=43: 25 \mathrm{~m}, 18 \mathrm{f}$,

3-77 years)

total mutation load in skeletal

muscle bulk mtDNA

$$
0 \% \quad 69 \pm 3 \% \quad 75 \pm 4 \%
$$

citrate synthase (CS)

$13.9 \pm 4.5$

6.7

10.0

NADH:CoQ1 reductase

(complex I)
$1.42 \pm 0.82$
0.09
0.68

complex I / CS

$0.11 \pm 0.045$

0.01

0.068

cytochrome coxidase

(complex IV)

Patient A with CPEO harbouring the novel G5835A tRNA ${ }^{\text {Tyr }}$, del4315A tRNA ${ }^{l l e}$, and T1658C tRNA ${ }^{\text {val }}$ mtDNA mutations.

Patient B with MELAS harbouring the A3243G tRNA ${ }^{\text {Leu(UUR) }}$ mtDNA mutation.

CS, citrate synthase; m, male f, female; wwt, wet weight; U, Units; g, gramme 


\section{Fig. 1}

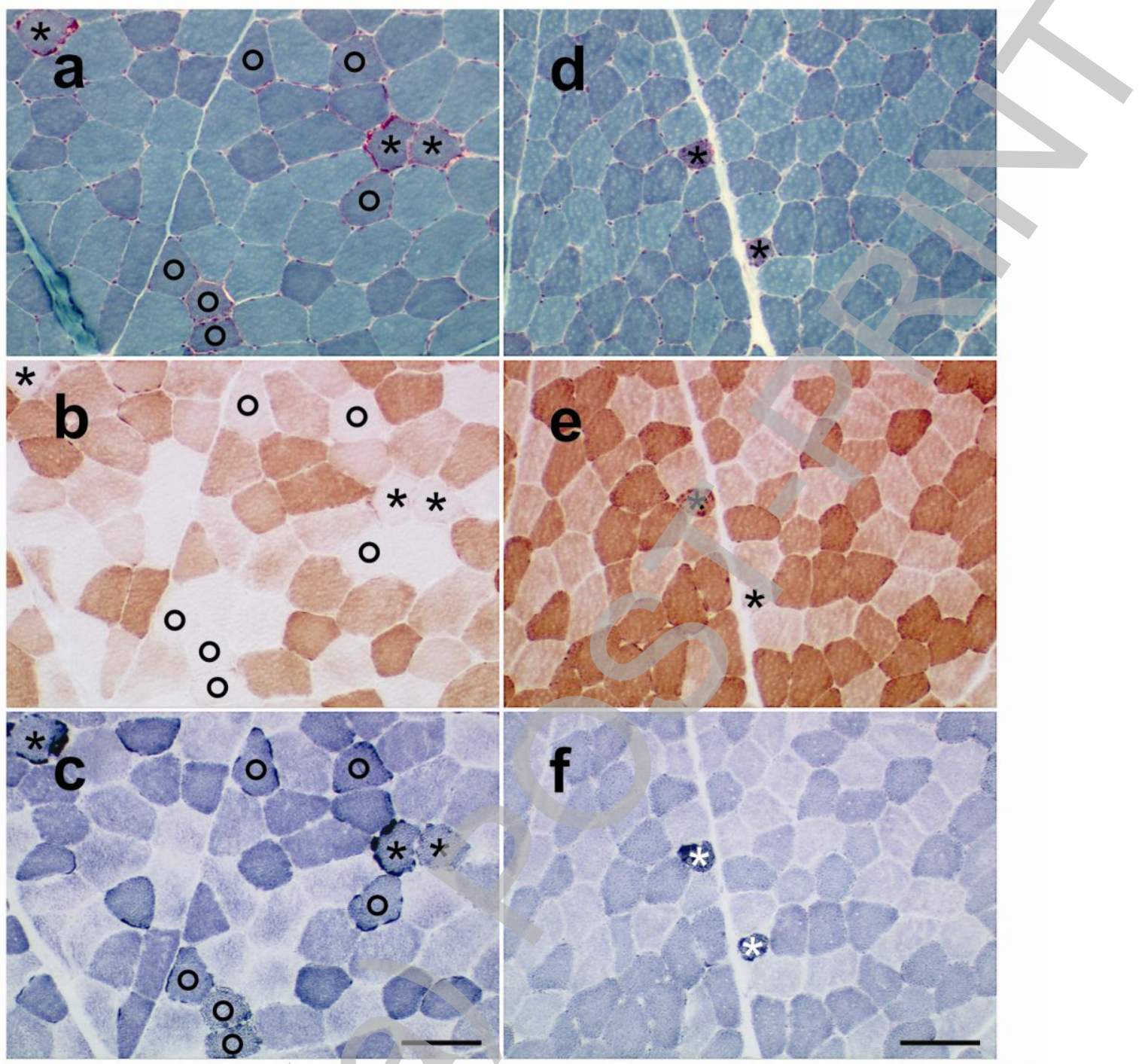

Histochemical stainings of serial sections of skeletal muscle biopsies of patients A (left figures, a-c) and B (right figures, $\mathbf{d}-\mathbf{f}$ ) developed under identical conditions.

(a, d) Modified Gomori's trichrome staining.

(b, e) Cytochrome coxidase (COX) activity. Notice the reduced overall activity as well as the mosaic pattern in COX staining of patient $A$.

$(\mathbf{c}, \mathbf{f})$ Succinate dehydrogenase (SDH) activity.

Star, ragged red fiber; circle, SDH-positive COX-negative fiber; scale bar, $100 \mu \mathrm{m}$. 


\section{Fig. 2}

a
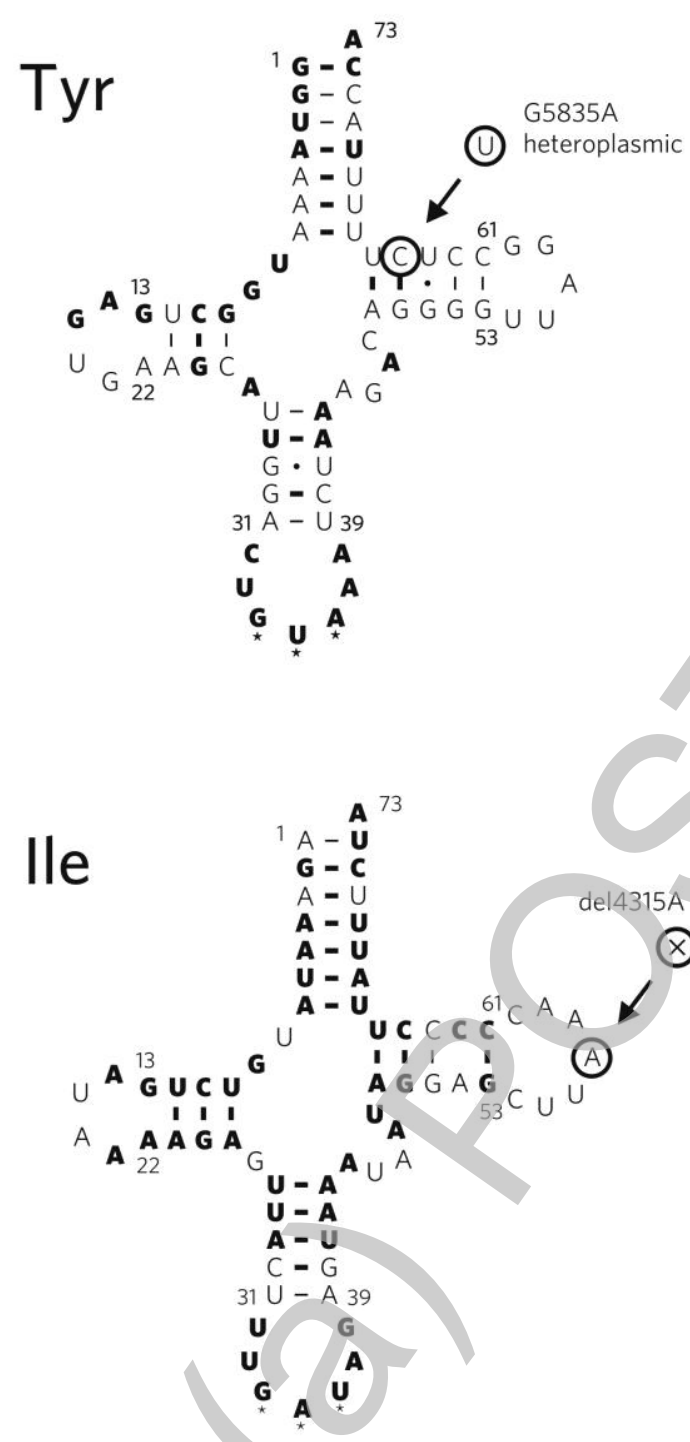

C

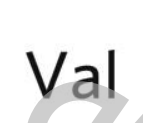

${ }^{1} \mathbf{C}-\mathbf{A}^{73}$

$A=U$
$G=C$
$A-U$

$A-U$

$G \cdot C$
$G \cdot C$

$u^{\mathrm{G}-\mathrm{C}}$
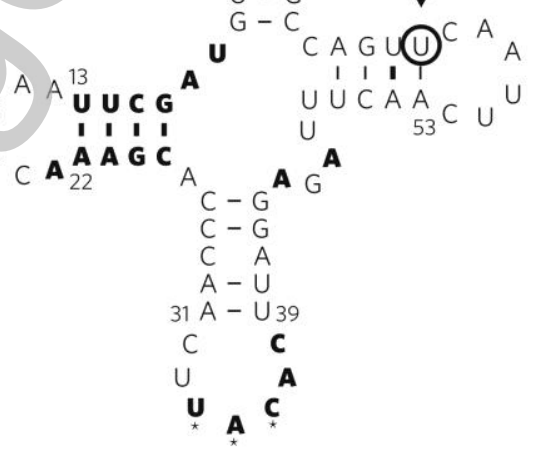

Licenced copy. Copying is not permitted, except with prior permission and as allowed by law. 
Three novel tRNA mtDNA mutations residing in the tRNA ${ }^{\text {Tyr }}$, $t R N A^{\text {lle }}$, and tRNA ${ }^{\text {Val }}$ genes of patient $A$. Conserved nucleotide positions and pairs are indicated by bold letters and thick lines, respectively (according to MAMIT-tRNA, http://mamit-tRNA.u-strasbg.fr). Watson-Crick nucleotide pairing is shown by lines, G-U pairing by dots. The anticodons are indicated by stars. Mutated positions are encircled and labelled by their genomic positions. tRNA position numbering follows conventional rules [19].

a) The mitochondrial G5835A tRNA ${ }^{\text {Tyr }}$ gene mutation results in a $\mathrm{C} \rightarrow \mathrm{U}$ transition at nucleotide 64 in the T-stem of the typical cloverleaf secondary structure of the mitochondrial tRNA ${ }^{\text {Tyr }}$. The mutation disrupts the classical Watson-Crick base pairing that is present in all sequenced mammals at this position [20]. The observed T-stem $\mathrm{C} \rightarrow \mathrm{U}$ transition is absent in all known mammalian mitochondrial tRNA ${ }^{\mathrm{Ty}}$ sequences with one exception (Dasypus novemcinctus, nine-banded armadillo). However, in this species a $\mathrm{G} \rightarrow \mathrm{A}$ transition of the complementary nucleotide is present thus maintaining the classical Watson-Crick base pairing. The human tRNA ${ }^{\text {Tyr }}$ T-stem regularly harbours a nonconventional $G-U$ base pair at nucleotides 51-63 (MAMIT-tRNA, Homo sapiens). The G5835A tRNA gene mutation thus results in a second G-U pairing in the T-stem at the adjacent position presumably resulting in lower stability of the tRNA ${ }^{\text {Tyr }}$ structure.

b) del4315A in the mitochondrial tRNA ${ }^{\text {Ile }}$ gene affects the tRNA T $\psi \mathrm{C}$ loop. The nucleotide composition of the tRNA ${ }^{\text {lle }} T \psi C$ loop is highly variable among species, whereas its size is moderately conserved at least among mammals. In the majority of mammals including primates, the number of nucleotides in the tRNA ${ }^{\text {lle }} T \psi \mathrm{C}$ loop is fixed to 7 [21, www.ncbi.nlm.nih.gov/genomes/ORGANELLES/mitoabout.html, mitochondrial genomes of 143 mammals], however its size may vary from 4 to 8 nucleotides. A 6-nucleotide tRNA ${ }^{\text {lle }}$ $\mathrm{T} \psi \mathrm{C}$ loop similar to our patient is present in some mammalian species belonging to various orders like Cetacea, Insectivora, or Artiodactyla.

c) The mitochondrial T1658C tRNA ${ }^{\text {Val }}$ gene mutation results in a $\mathrm{U} \rightarrow \mathrm{C}$ transition at nucleotide 61 in the T-stem of the mitochondrial tRNA ${ }^{\mathrm{Val}}$ at an evolutionary not well conserved position. The mutation can be found in various mammalian species also comprising primates, e.g. Pongo pygmaeus (orangutan). 


\section{Fig. 3}

a

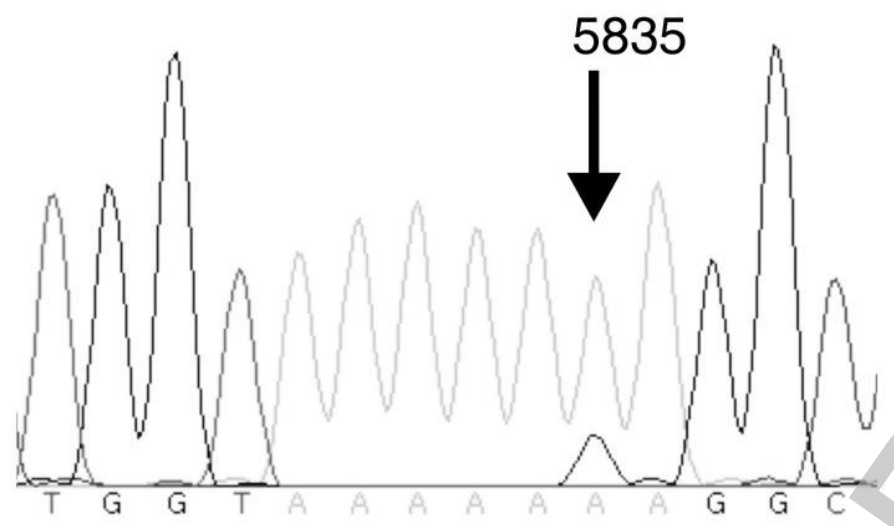

b
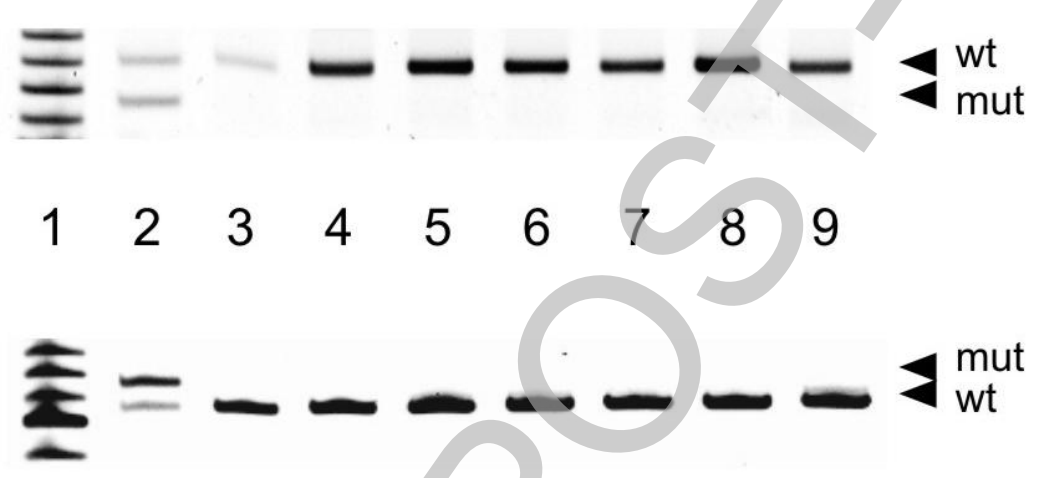

$\begin{array}{lllllllll}1 & 2 & 3 & 4 & 5 & 6 & 7 & 8 & 9\end{array}$

Quantification of the heteroplasmic G5835A tRNA ${ }^{\text {Tyr }}$ mtDNA mutation.

a) Partial sequence of the tRNA ${ }^{\text {Tyr }}$ amplified from the patient's skeletal muscle bulk DNA.

b) Proportions of the mutant G5835A allele in various tissues from the patient and four relatives, analysed by digestion of the mutant (mut) allele

c) Proportions of the mutant G5835A allele in various tissues from the patient and four relatives, analysed by digestion of the wild-type (wt) allele.

Molecular weight marker (lane 1), patient's skeletal muscle (lane 2), patient's buccal mucosa (lane 3), patient's blood (lane 4), sister A's blood (lane 5), sister B's blood (lane 6), brother's blood (lane 7), mother's blood (lane 8), control skeletal muscle (lane 9). 
Fig. 4

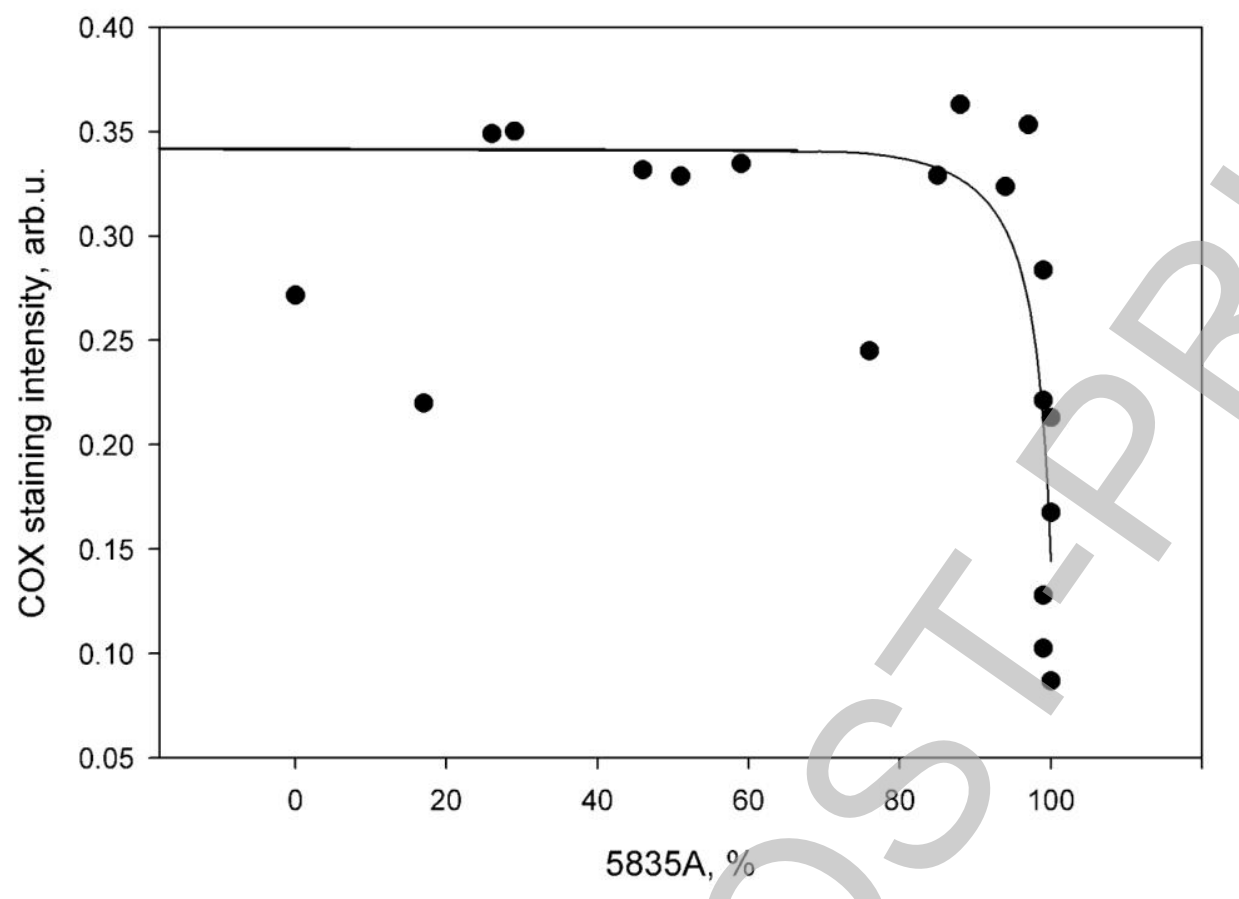

Correlation of COX activities with G5835A tRNA ${ }^{\text {Tyr }}$ mtDNA mutation loads in single skeletal muscle fibers of patient $A$ (each data point represents a single type I skeletal muscle fiber). Note the high threshold for phenotypic expression of this mutation. 
Fig. 5

a
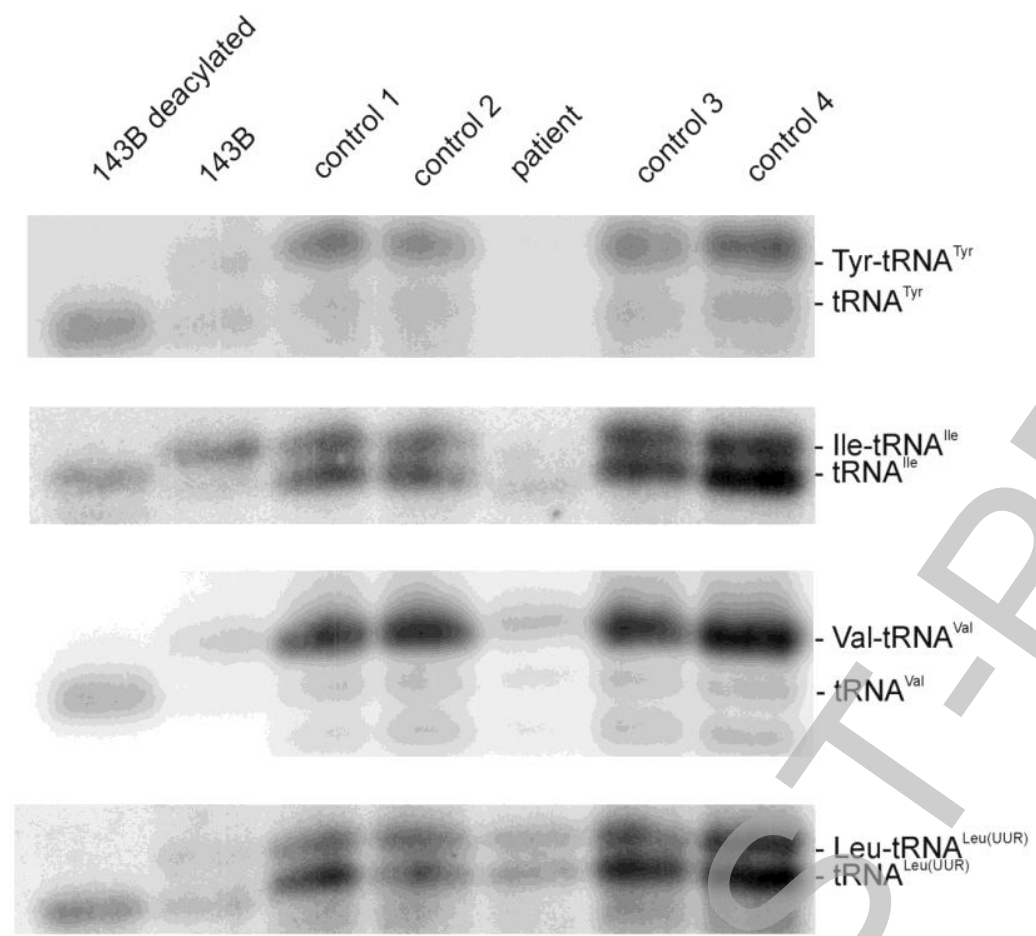

b
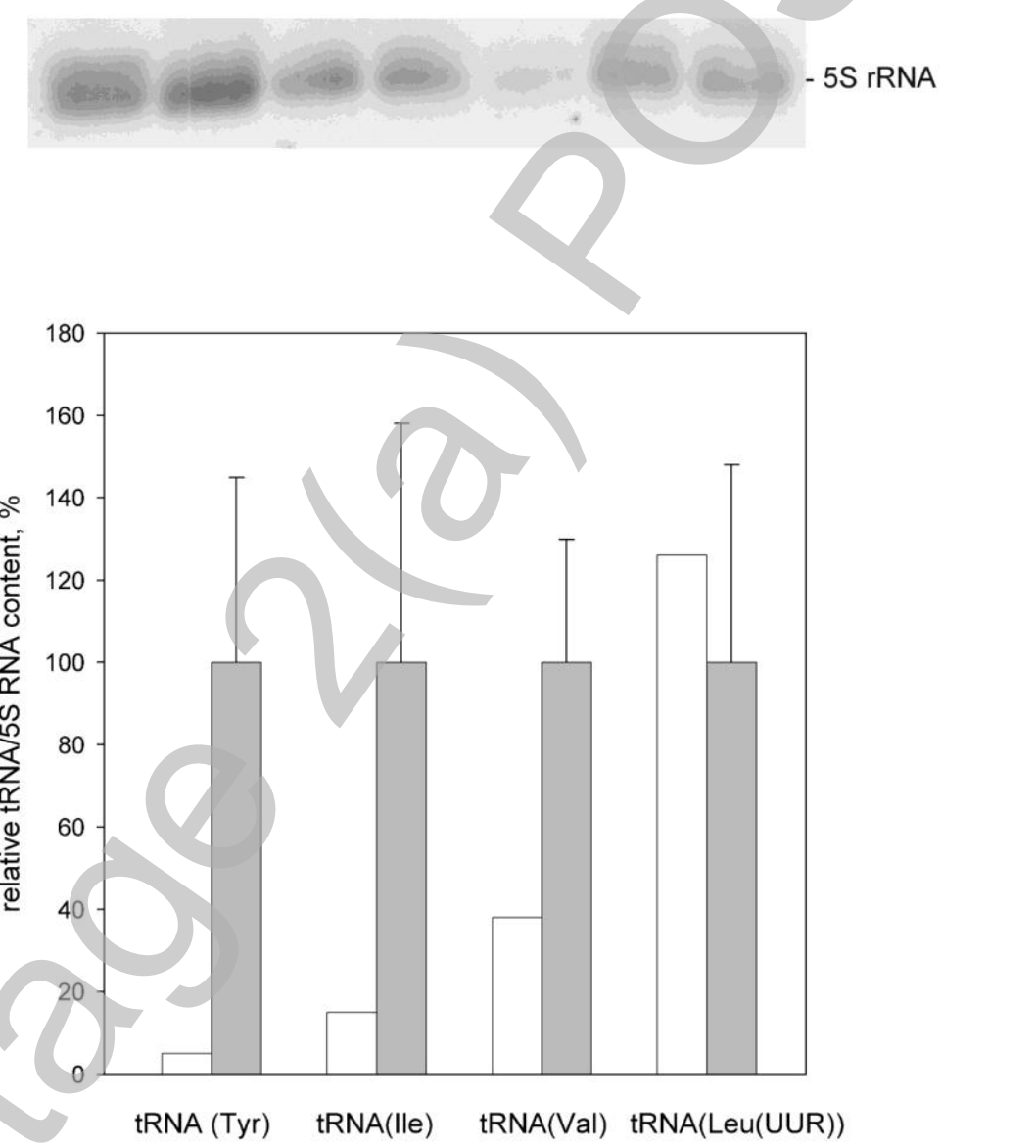
Steady state tRNA levels in the patient's skeletal muscle.

a) Northern blot. RNA from 143B osteosarcoma cells and from muscle biopsies of patient $A$ and controls was isolated and run under acidic denaturing conditions, blotted and probed for tRNA $^{\text {Tyr }}$, tRNA ${ }^{\text {Ile }}$, tRNA ${ }^{\text {Val }}$, tRNA ${ }^{\text {Leu(UUR) }}$, and for $5 S$ rRNA. A sample of deacylated RNA from 143B cells obtained by alkaline treatment was also loaded for comparison.

b) tRNA / 5S rRNA ratios in the skeletal muscle of the patient (white bars) and of four control skeletal muscle biopsies (grey bars). Values for tRNAs are the sum of signals for

aminoacylated and deacylated form. The control data (grey bars) are expressed as mean \pm SD of four biopsies; all data were analysed using a phosphoimager. 